\title{
Control of interparticle spacing in stable aggregates of gold nanoparticles by light irradiation
}

\author{
Kazuo Tanaka ${ }^{1}, K_{\text {Kensuke Naka }}{ }^{2}$, Eisuke Miyoshi ${ }^{1}$, Asako Narita ${ }^{1}$ and Yoshiki Chujo ${ }^{1}$ \\ This study describes the modulation of interparticle distances in aggregates of gold nanoparticles by light irradiation. Stable \\ aggregates of a series of imidazolium-presenting gold nanoparticles were obtained via a photo-responsive mono-carboxylate \\ linker. In the first step, the mono-carboxylate linker attracted the gold nanoparticles to form water-dispersive aggregates of gold \\ nanoparticles with hydrophobic surface properties. By irradiating the aggregates with ultraviolet, the photo-responsive linker was \\ transformed, leading to the generation of another carboxyl group. As a result, the gold nanoparticles were tightly bound via the \\ dicarboxylate linkers. These changes decreased the interparticle distances in the aggregates, as verified by microscopic \\ observations, and can induce significant changes in their optical characteristics. To the best of our knowledge, this is the first \\ example of a light-driven manipulation system for the distribution of nanoparticles in an aggregate. \\ Polymer Journal (2015) 47, 747-752; doi:10.1038/pj.2015.56; published online 5 August 2015
}

\section{INTRODUCTION}

The physical properties of metal nanoparticles strongly depend on their assembled states. It is known that an assembly of gold nanoparticles has different light-absorption properties to that of single particles. ${ }^{1,2}$ Based on this phenomenon, a wide variety of biomedical applications has been accomplished. ${ }^{3,4}$ In addition, by manipulating the hybridization process involved in anchoring DNA to the surface of particles, cluster formation and morphology can be precisely controlled. ${ }^{5-7}$ These manipulation methods for the nanoparticle assembly are versatile and can be used to construct biosensors with good response to stimuli. ${ }^{8,9}$ Furthermore, additional functions have been derived from the specific distribution of nanoparticles in an assembly. For instance, a class of unique optical functional materials involving metamaterials has been manufactured with a well-ordered assembly of gold nanoparticles. ${ }^{10-13}$ Therefore, precisely controlling the morphology and relative positions of nanoparticles in an assembly is critically important to obtain superior or unexpected functions. ${ }^{14-20}$

Structural manipulation with light has various advantages. A rapid, time-sensitive, and site-sensitive response can be achieved without additives. In previous reports, the photoisomerizations of azobenzene $e^{21-26}$ and stilbene derivatives ${ }^{27-29}$ tethered to the surface of nanoparticles have been investigated. Based on the structural changes triggered by the light irradiation, the dispersion states were controlled. The aggregation/dispersion of gold nanoparticles can also be modulated reversibly by the photo-triggered dimerization of thymine and the splitting of the thymine dimer. ${ }^{30}$ Although the formation of aggregates can be controlled with various methods, as mentioned here, modulating the morphology of the aggregates is less well established.
In particular, few examples have controlled the interparticle distances in the nanoparticle aggregates. We have previously demonstrated that imidazolium-presenting gold nanoparticles can form stable and welldispersed aggregates in aqueous solutions. ${ }^{31}$ The hydrophobicity of the surface was modulated via the anion exchange of the imidazolium cation at the surface of the nanoparticles. Finally, the interparticle distances of the gold nanoparticles in the aggregates could be regulated at the nanometer scale. Accordingly, the light-absorption properties in the visible region originating from the surface plasmon resonance of the gold nanoparticles could be tuned under mild conditions. The next challenge is to construct a modulation system for the distances between the gold nanoparticles in the aggregate by external stimuli, such as light irradiation.

Here we report the modulation of the interparticle distances in aggregates of gold nanoparticles via light irradiation. Modified nanoparticles covered with an imidazolium salt were prepared and mixed with a carboxylate-containing linker that has a photo-cleavable $o$-nitrobenzyl ester. In the first step, the linker molecules gathered the synthesized nanoparticles to form a stable and well-dispersed aggregate under aqueous conditions. Triggered by ultraviolet (UV) irradiation, the o-nitrobenzyl ester in the linker was cleaved, resulting in the generation of another carboxylate group. Finally, the nanoparticles were tightly bound by the dicarboxylate linkers. These changes can be monitored by microscopic observations. In addition, significant changes were induced in the optical properties of the gold nanoparticles. To the best of our knowledge, this is the first example of a lightdriven modulation system for the distribution of nanoparticles in an aggregate.

${ }^{1}$ Department of Polymer Chemistry, Graduate School of Engineering, Kyoto University, Kyoto, Japan and ${ }^{2}$ Faculty of Molecular Chemistry and Engineering, Graduate School of Science and Technology, Kyoto Institute of Technology, Kyoto, Japan

Correspondence: Professor Y Chujo, Department of Polymer Chemistry, Graduate School of Engineering, Kyoto University, Katsura, Nishikyo-ku, Kyoto 615-8510, Japan. E-mail: chujo@chujo.synchem.kyoto-u.ac.jp

Received 5 April 2015; revised 10 June 2015; accepted 20 June 2015; published online 5 August 2015 


\section{EXPERIMENTAL PROCEDURE}

\section{General}

All of the reactions were conducted under a nitrogen atmosphere unless otherwise stated. Chromatographic purifications were performed using Wako gel C-200 (Wako Pure Chemical Industries, Ltd., Osaka, Japan). An ultrafilter unit (USY-5, molecular weight cutoff $=50000$ ) was purchased from ADVANTEC Co., Ltd (Tokyo, Japan). ${ }^{1} \mathrm{H}$ and ${ }^{13} \mathrm{C}$ nuclear magnetic resonance (NMR) spectra were obtained with a JEOL EX-400 spectrometer ( $400 \mathrm{MHz}$; JEOL Ltd, Tokyo, Japan) using chloroform- $d_{1}$, deuterium oxide and dimethylsulfoxide- $d_{6}$ as solvents and either tetramethylsilane or trimethylsilylpropionic acid sodium salt as an internal reference. UV-visible spectra were measured on a SHIMADZU UV-3600 spectrophotometer using quartz cuvettes with a $1-\mathrm{cm}$ optical path length (SHIMADZU CORPORATION, Kyoto, Japan). Transmission electron microscopy (TEM) was performed using a JEOL JEM-1025 operated at a $100-\mathrm{kV}$ electron beam accelerating voltage (JEOL Ltd). One drop of sample was deposited onto a carbon grid, the excess liquid was blotted off with a Kimwipe and the grid was dried under ambient conditions. Dynamic light scattering was measured on a FPAR-1000 (Otsuka Electronics Co., Ltd, Osaka, Japan) at $20.0^{\circ} \mathrm{C}$, using the Marquardt method to solve the autocorrelation functions. The samples were irradiated with UV light with a spiralshaped low-pressure mercury lamp. The samples were added to a quartz-glass tube and were placed at the center of the spiral-shaped lamp. Carboxylate $1^{32}$ and imidazolium-presenting gold nanoparticles NP1-3 $3^{31}$ with various degrees of hydrophobicity were prepared according to previous reports.

\section{Preparation of the gold nanoparticle aggregates}

To the aqueous dispersion of nanoparticles $\left(5 \mathrm{ml}, 4 \times 10^{-4} \mathrm{~mol}\right.$ of imidazolium), $1 \mathrm{ml}$ of $0.04 \mathrm{M}$ aqueous solution of 1 was added and left for $1 \mathrm{~h}$, followed by washing with $1 \mathrm{ml}$ of water five times using an ultrafilter unit (ADVANTEC USY-5, molecular weight cutoff $=50000$ ). Then the nanoparticles were dispersed in $6 \mathrm{ml}$ of distilled water and measured.

\section{Photoreactions}

A typical procedure involved the following steps: the sample dispersion $(3 \mathrm{ml})$ was added to a quartz-glass test tube, which was located at the center of the spiral-shaped mercury lamp. Then the UV irradiation was performed at room temperature.

\section{RESULTS AND DISCUSSION}

The synthesis of the gold nanoparticle aggregates and the schematic model of the regulation of the interparticle distances are illustrated in
Scheme 1. To form a water-dispersive aggregate of gold nanoparticles, the carboxylate anion 1, which has a photo-cleavable functional group, was mixed into the imidazolium-presenting gold nanoparticles by employing ionic liquid structures ${ }^{33,34}$ via anion exchange with chloride anions. Under aqueous conditions, the gold nanoparticles should be aggregated because of the enhanced hydrophobicity due to 1 . Using light irradiation, another carboxyl group could be generated, followed by an interaction with the neighbor imidazolium cation. Following this procedure, the nanoparticles are expected to be tightly bound. Compound 1 was synthesized according to a previous study. ${ }^{32}$ The sodium form of $\mathbf{1}$ was obtained as a pale yellow solid with a cation exchange resin. Modified gold nanoparticles with the chloride form of imidazolium were prepared according to our previous report. ${ }^{31}$ To examine the influence of surface hydrophobicity on the behavior of the aggregates, three types of nanoparticles were prepared with various substituent structures in imidazolium. NP1 has the most hydrophilic surface of the three, and NP3 has the most hydrophobic and bulky imidazolium moiety substituent. The anion exchange from chloride anions to carboxylate anions was examined using ${ }^{1} \mathrm{H}$ NMR measurements (Supplementary Figure $\mathrm{S} 1$ ). To $\mathrm{D}_{2} \mathrm{O}$ solutions of imidazolium, 10 eq. of 1 was added and incubated for $60 \mathrm{~min}$ at room temperature. Then ${ }^{1} \mathrm{H}$ NMR measurements were performed. The peak position of the NMR signal derived from the proton at the 2-position in the imidazolium ring was shifted from 8.55 to 9.74 p.p.m. by adding 1 . This result suggests that anion exchange occurred.

Initially, the aggregation behaviors of the nanoparticles were investigated. Figure 1 shows representative TEM images of NP2 before and after adding $\mathbf{1}$. Well-dispersed states were obtained from all of the dispersions of the synthesized nanoparticles before adding $1 .^{31}$ By contrast, aggregate states were clearly induced in the dispersions, especially those containing NP2 and NP3, which possess relatively hydrophobic alkyl groups in the imidazolium moiety in the presence of 1. From the sample containing NP1, which has the most hydrophilic modification of the three, aggregates were partially formed. The interparticle distances were estimated as an averaged value of the distance between the centers of the particles from the TEM observations. Table 1 presents the averaged interparticle distances between the nanoparticles in the aggregates. It is likely that

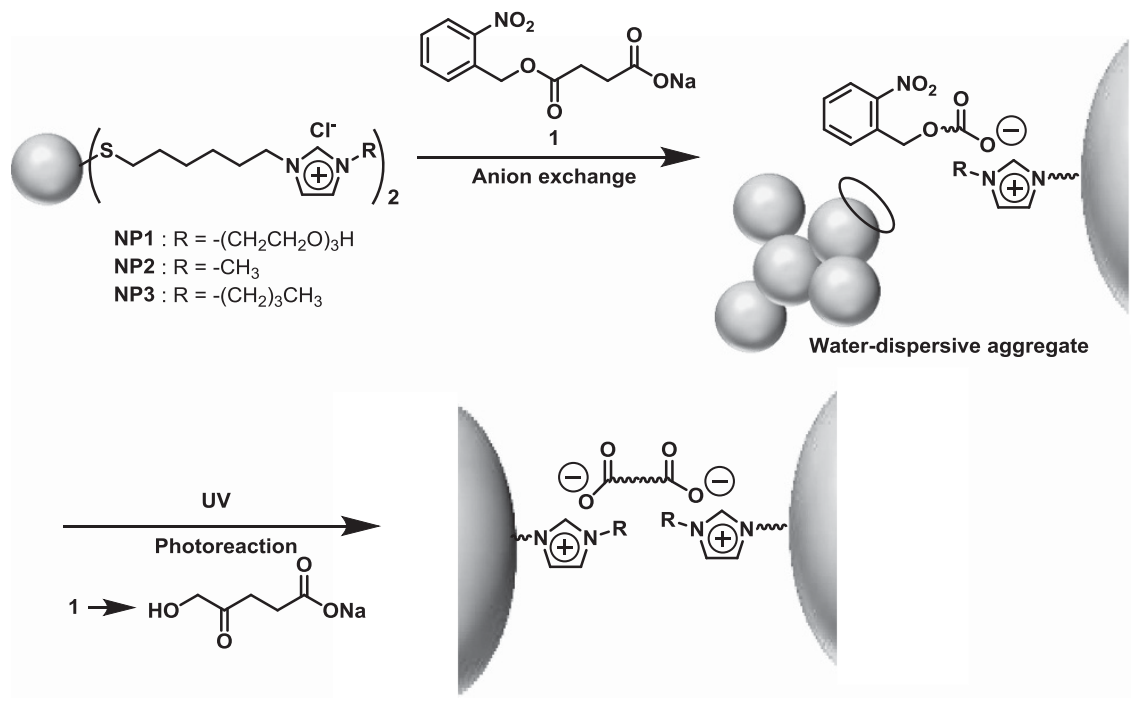

Scheme 1 Schematic illustration of the modulation of interparticle distances with the photo-responsive linker 1 . A full color version of this scheme is available at the Polymer Journal online. 

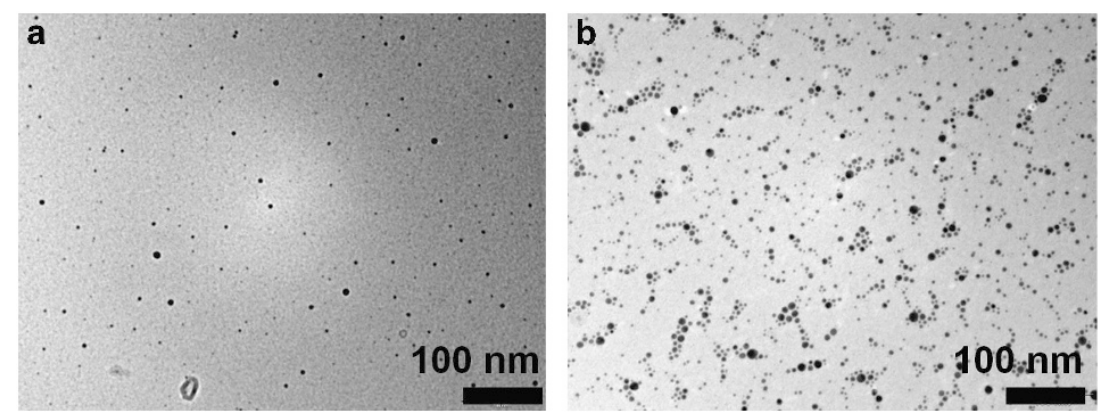

Figure 1 TEM images of NP2 (a) before and (b) after addition of 1.

Table 1 Summary of absorption maxima and the interparticle distances of the aggregates of the modified gold nanoparticles ${ }^{a}$

\begin{tabular}{|c|c|c|c|c|c|c|}
\hline & \multicolumn{2}{|c|}{$N P 1$} & \multicolumn{2}{|c|}{$N P 2$} & \multicolumn{2}{|r|}{ NP3 } \\
\hline & $\lambda_{\max }(n m)$ & $\begin{array}{l}\text { Interparticle distance } \\
\qquad(n m \pm \text { s.d. })^{\mathrm{b}}\end{array}$ & $\lambda_{\max }(n m)$ & $\begin{array}{l}\text { Interparticle distance } \\
\qquad(n m \pm \text { s.d. })^{b}\end{array}$ & $\lambda_{\max }(n m)$ & $\begin{array}{c}\text { Interparticle distance } \\
(n m \pm \text { s.d. })^{\mathrm{b}}\end{array}$ \\
\hline After preparation & 512 & $-^{c}$ & 515 & $-^{c}$ & 512 & $-^{c}$ \\
\hline After 1 addition & 513 & - & 522 & $2.2(0.6)$ & 575 & $2.0(0.6)$ \\
\hline After UV irradiation ${ }^{d}$ & 540 & $1.7(0.6)$ & 552 & $1.6(0.4)$ & 580 & $1.2(0.6)$ \\
\hline
\end{tabular}

Abbreviation: UV, ultraviolet.

aMeasured at room temperature.

Determined from the transmission electron microscopic measurements.

cAggregation was not observed.

drradiated for $10 \mathrm{~min}$ at room temperature.

the nanoparticles with longer alkyl chains can aggregate because of their strong hydrophobicities.

It is known that the light-absorption ability of gold nanoparticles can indicate the dispersion state of the nanoparticles. ${ }^{31}$ By forming aggregates, the absorption band can be shifted to a longer-wavelength region. Correspondingly, as a representative result, an absorption band was observed with a peak at $512 \mathrm{~nm}$ before adding 1 to the dispersion of NP2 (Figure 2). By contrast, a red-shifted absorption band was observed with a peak at $522 \mathrm{~nm}$. With the NP3 nanoparticles, a peak shift to the longer-wavelength region was also induced by adding $\mathbf{1}$. These data clearly indicate that carboxylate 1 can enhance the formation of aggregates. Furthermore, from the dynamic light scattering measurements, an increase of the hydrodynamic radii from $5.4 \pm 1.4$ to $11.3 \pm 2.0 \mathrm{~nm}$ was observed by adding 1 to the dispersion of NP2 nanoparticles. These data also support the formation of welldispersed synthesized nanoparticle aggregates.

Before photoreactions with the synthesized nanoparticles, we examined the reactivity of $\mathbf{1}$ and determined the products after light irradiation. Because of the absorption maximum of 1 at $266 \mathrm{~nm}$, a low-pressure mercury lamp (>300 nm) was used to induce a photocleavage reaction. To $5 \mathrm{ml}$ of a $\mathrm{D}_{2} \mathrm{O}$ solution of $1(0.01 \mathrm{M})$, UV light was irradiated through quartz glass, and the reactions were monitored using ${ }^{1} \mathrm{H}$ NMR (Figure 3). After photo-irradiating the solution of $\mathbf{1}$, the signal intensity derived from the aromatic ring in $\mathbf{2}$ decreased with the photo-irradiation time, whereas the signal intensity around $\delta 2.48$ p.p.m., derived from monosodium succinate, increased. These data indicate the progress of the photo-cleavage leading to the generation of the carboxyl group. By evaluating the conversion, $80 \%$ of 1 was determined to be consumed within $30 \mathrm{~min}$. The spectra showed that less by-product was generated by UV irradiation. By contrast, by increasing the irradiation time, the consumption yield of 1 was improved. However, unexpected side-reactions were observed.
The photo-induced cleavage of $\mathbf{1}$ was also monitored with UV-vis measurements (Figure 4). UV light from a low-pressure mercury lamp irradiated a $3.3 \times 10^{-5} \mathrm{M}$ aqueous solution of 1 through quartz glass, and changes in absorptions were recorded using a UV-vis spectrometer. Photo-irradiation to $\mathbf{1}$ caused the decrease of the absorption band at $266 \mathrm{~nm}$. These data indicate the generation of $o$-nitrosobenzaldehyde. ${ }^{35,36}$ In other words, the data support the production of a carboxyl group.

The photoreaction was carried out with the same light source at room temperature for $10 \mathrm{~min}$. From the TEM observations, the interparticle distances were evaluated (Figure 5). The UV irradiation decreased the interparticle distances. The averaged diameter of NP2 decreased from $2.2 \pm 0.7$ to $1.6 \pm 0.5 \mathrm{~nm}$. From the dynamic light scattering measurements, a drastic increase in the hydrodynamic radii, from $11.3 \pm 2.0$ to $618 \pm 403 \mathrm{~nm}$, was observed. These data clearly indicate that UV irradiation can induce tight binding in the aggregates. Even from the dispersion of NP1, which was too well dispersed to determine the interparticle distances in the presence of $\mathbf{1}$, aggregates with an interparticle distance of $1.6 \pm 0.5 \mathrm{~nm}$ were observed. Thus the data show that the product of $\mathbf{1}$ after UV irradiation has a superior nanoparticle aggregation property.

The optical properties were greatly influenced by the UV irradiation (Figure 2 and Supplementary Figure S2). After the UV irradiation of the dispersion, the absorption band with the 266-nm peak decreased. It was hypothesized that the photo-cleavable group would be removed, leading to the generation of carboxylates. Significantly, UV irradiation caused a red-shift in the surface plasmon absorption. Peak shifts by +37 nm (NP1), +30 nm (NP2) and +5 nm (NP3) after UV irradiation were observed in the dispersions. The relatively smaller degree of redshift in NP3 after UV irradiation could be derived from the intrinsic tight binding among the nanoparticles. Because of the strong hydrophobicity of the alkyl chains in NP3, aggregation could be induced 

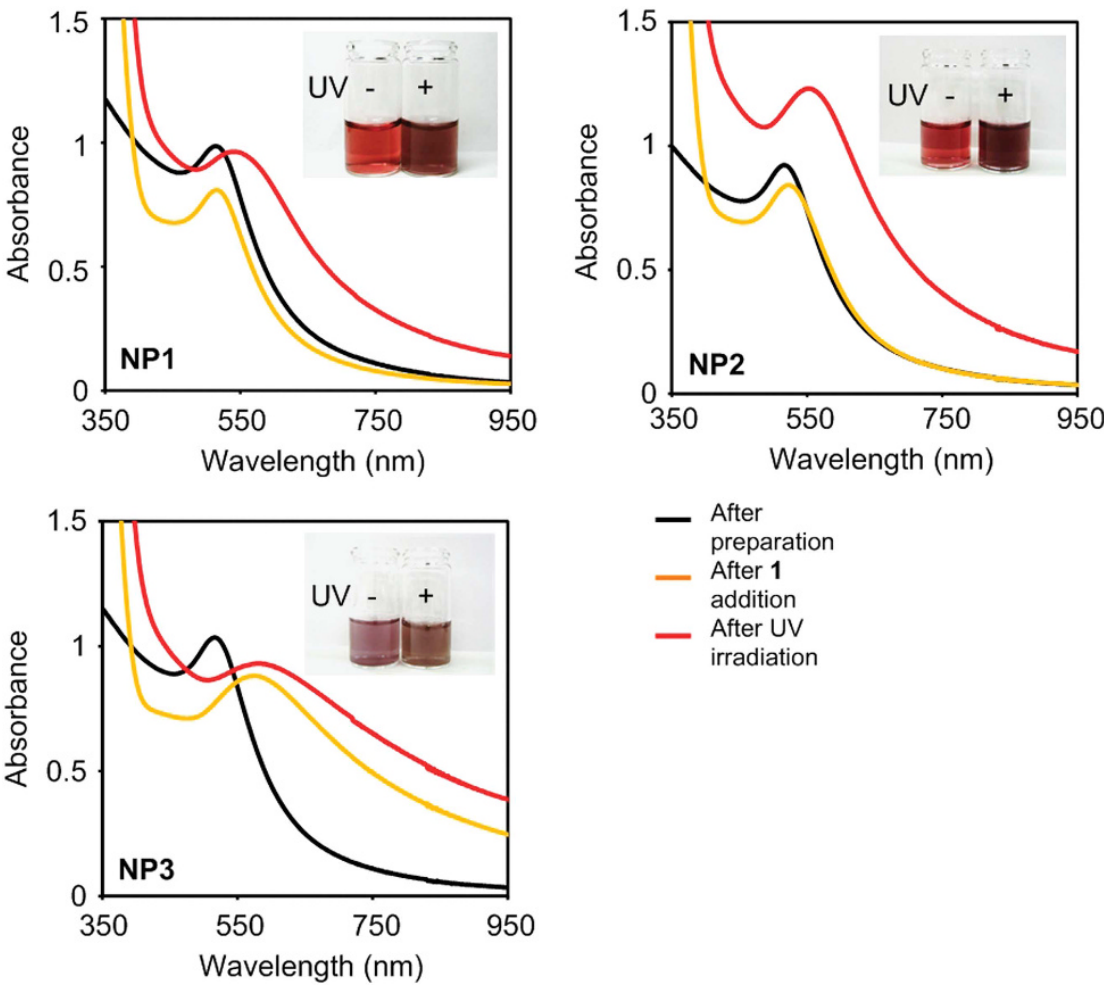

Figure 2 UV absorption spectra of the nanoparticles in aqueous dispersions at room temperature. The pictures show the appearances of the dispersions before and after UV irradiation for $10 \mathrm{~min}$.

a

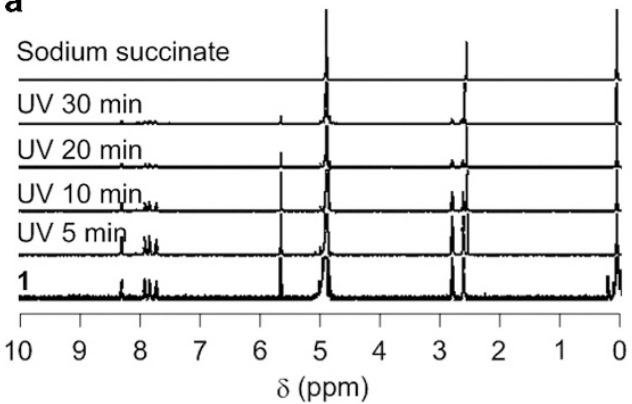

b

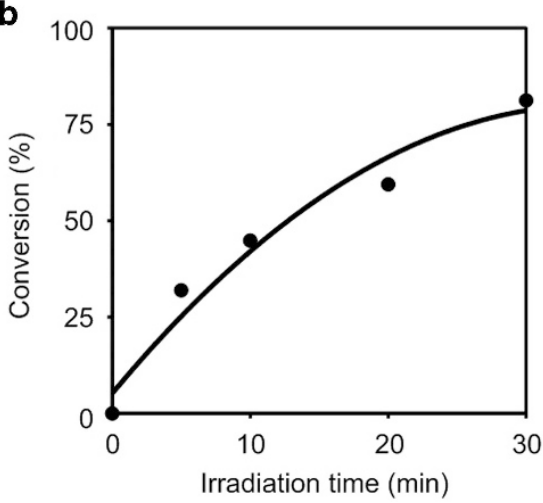

Figure 3 (a) NMR spectra of sodium succinate and $1(0.01 \mathrm{~m})$ before and after low-pressure mercury lamp irradiation (in $\mathrm{D}_{2} \mathrm{O}, 400 \mathrm{MHz}$, with trimethylsilylpropionic acid sodium salt used as an internal standard). (b) Plot of conversion vs irradiation time. The conversions were calculated from the ratio of the NMR signal intensities observed at 2.41 and 5.54 p.p.m. only by adding compound 1 . The conversion after $10 \mathrm{~min}$ of irradiation was calculated to be $69 \%$. After longer irradiations, the consumption rate increased to $80 \%$ after a 20 -min irradiation and finally $100 \%$ after a 30 -min irradiation.

In the absence of $\mathbf{1}$, the absorption property of the well-dispersed NP2 was not strongly influenced by the light irradiation (Supplementary Figure S3). In addition, to prove the necessity of a photo-responsive anion, we conducted the same experiment with sodium benzoate instead of $\mathbf{1}$ (Supplementary Figure S4). Similar to 1, sodium benzoate also induced the aggregation of NP2, and the redshifted absorption with the $520 \mathrm{~nm}$ peak was observed. However, significantly less red-shift was observed after the UV irradiation of the sample. These data indicate that the photo-moiety in $\mathbf{1}$ is essential to operate this system.

\section{CONCLUSION}

Our results demonstrate two significant points. First, a simple tool for manipulating the distribution of nanoparticles after the formation of aggregates was developed. In addition, these changes can be detected as a significant alteration in the optical properties. Based on this method, advanced optical materials can be produced with unique characteristics originating from precisely controlled nanostructures. Second, we can control the distribution of the nano-sized components in the system with small molecules and light irradiation. Our materials can significantly absorb light in the near infrared region. Our materials could potentially improve the conversion efficiency of solar cells by enhancing their light absorption efficiency in the near infrared region. In addition, because photosynthesis in several types of algae can be enhanced or suppressed by light irradiation in the specific wavelength 
a

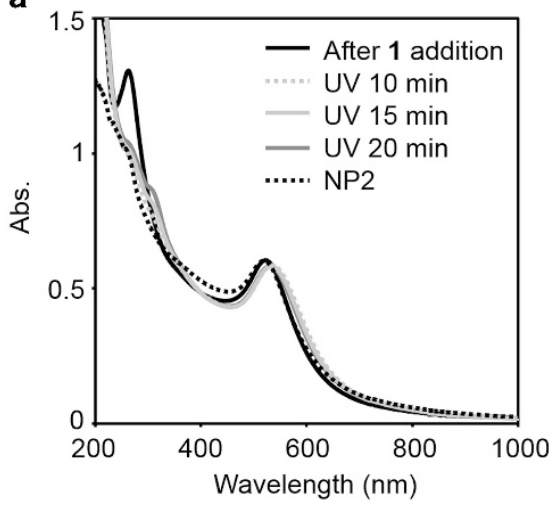

b
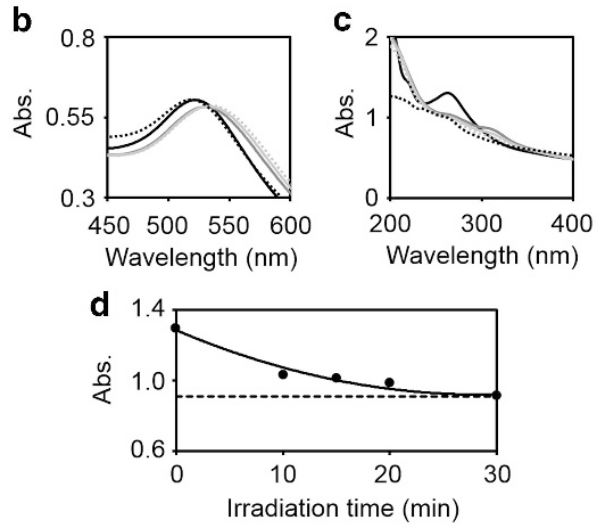

Figure 4 (a) Time course of the NP2 spectra in aqueous dispersion during UV irradiation. (b, c) Magnified spectra. (d) Time course of the absorption change at $266 \mathrm{~nm}$ during UV irradiation.
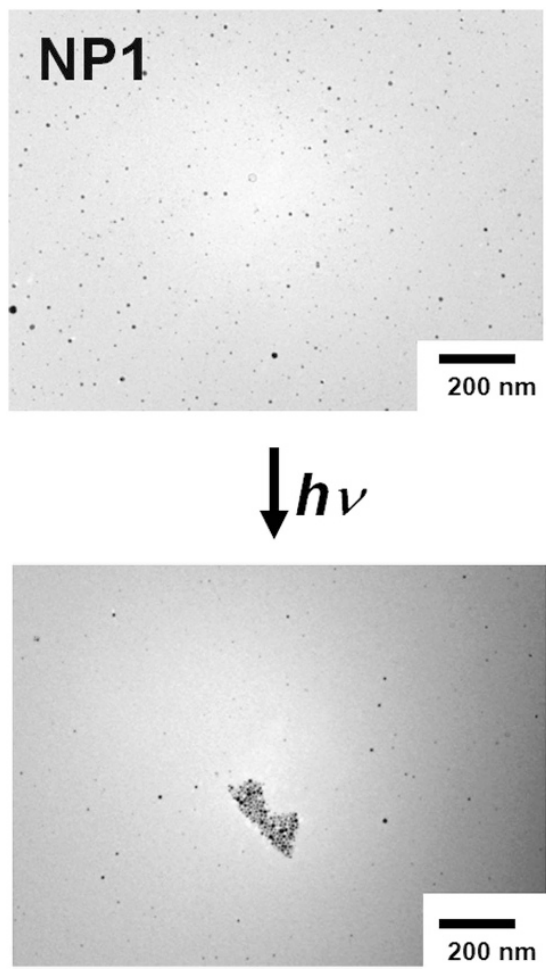
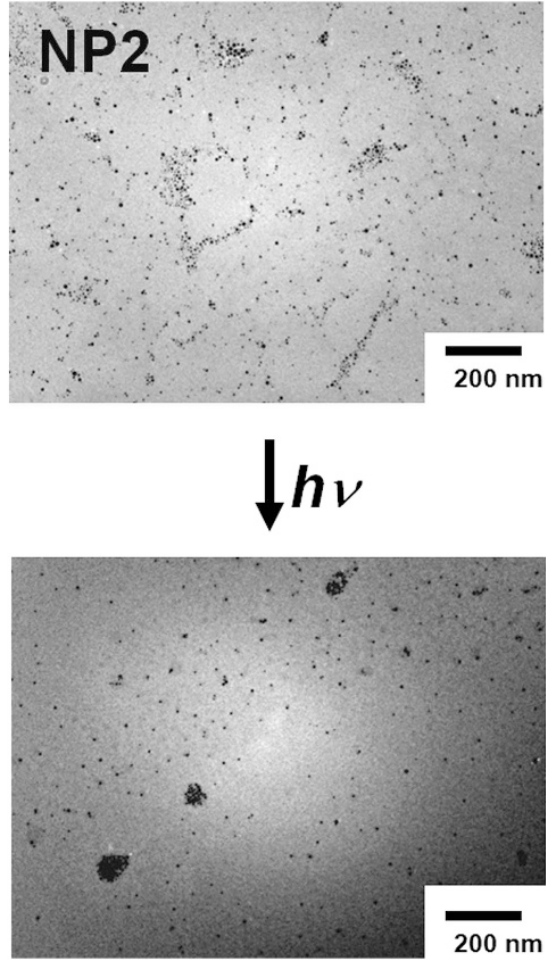
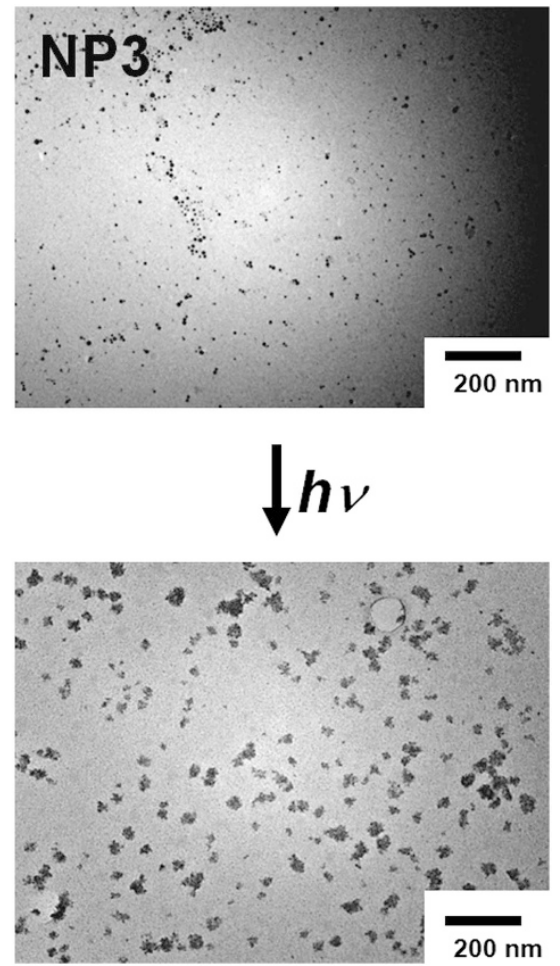

Figure 5 TEM images of (left) NP1, (middle) NP2, (right) NP3 after (upper) addition of 1 and (bottom) UV irradiation for 10 min. The scale bars represent $200 \mathrm{~nm}$.

range between the deep red and near infrared regions, materials that filter incident sunlight can be applied to plant growth. ${ }^{37}$ Our materials can be used as filtering materials for modulating plant growth. Thus our concept presented here is feasible not only for constructing nanostructured materials but also for dynamically regulating nanostructures.

\section{CONFLICT OF INTEREST}

The authors declare no conflict of interest.

\section{ACKNOWLEDGEMENTS}

This work was partially supported by Hosokawa Powder Technology

Foundation (to KT) and a Grant-in-Aid for Scientific Research on Innovative
Areas 'New Polymeric Materials Based on Element-Blocks (No.2401)' (25102521) from The Ministry of Education, Culture, Sports, Science, and Technology, Japan.

1 Daniel, M. C. \& Astruc, D. Gold nanoparticles: assembly, supramolecular chemistry, quantum-size-related properties, and applications toward biology, catalysis, and nanotechnology. Chem. Rev. 104, 293-346 (2004).

2 Ghosh, S. K. \& Pal, T. Interparticle coupling effect on the surface plasmon resonance of gold nanoparticles: from theory to applications. Chem. Rev. 107, 4797-4862 (2007).

3 Saha, K., Agasti, S. S., Kim, C., Li, X. \& Rotello, V. M. Gold nanoparticles in chemical and biological sensing. Chem. Rev. 112, 2379-2779 (2012).

4 Sapsford, K. E., Algar, W. R., Berti, L., Gemmill, K. B., Casey, B. J., Oh, E., Stewart, M. H. \& Medintz, I. L. Functionalizing nanoparticles with biological molecules: developing chemistries that facilitate nanotechnology. Chem. Rev. 113, 1904-2074 (2013). 
5 Mirkin, C. A., Letsinger, R. L., Mucic, R. C. \& Storhoff, J. J. A DNA-based method for rationally assembling nanoparticles into macroscopic materials. Nature $\mathbf{3 8 2}$, 607-609 (1996).

6 Šípová, H. \& Homola, J. Surface plasmon resonance sensing of nucleic acids: a review. Anal. Chim. Acta 773, 9-23 (2013).

7 Chen, J. I. L., Chen, Y. \& Ginger, D. S. Plasmonic nanoparticle dimers for optical sensing of DNA in complex media. J. Am. Chem. Soc. 132, 9600-9601 (2010).

8 Tanaka, K. \& Chujo, Y. Design of functionalized nanoparticles for the applications in nanobiotechnology. Adv. Powder Technol. 25, 101-113 (2014).

9 Kitamura, N., Tanaka, K. \& Chujo, Y. Heat-initiated detection for reduced glutathione with 19 F NMR probes based on modified gold nanoparticles. Bioorg. Med. Chem. Lett. 23, 281-286 (2013).

10 Kubo, S., Diaz, A., Tang, Y., Mayer, T. S., Khoo, I. C. \& Mallouk, T. E. Tunability of the refractive index of gold nanoparticle dispersions. Nano Lett. 7, 3418-3423 (2007).

11 Liu, Q., Cui, Y., Gardner, D., Li, X., He, S. \& Smalyukh, I. I. Self-alignment of plasmonic gold nanorods in reconfigurable anisotropic fluids for tunable bulk metamaterial applications. Nano Lett. 10, 1347-1353 (2010).

12 Moreau, A., Ciracì, C., Mock, J. J., Hill, R. T., Wang, Q., Wiley, B. J., Chilkoti, A. \& Smith, D. R. Controlled-reflectance surfaces with film-coupled colloidal nanoantennas. Nature 491, 86-89 (2012).

13 Pratibha, R., Park, K., Smalyukh, I. I. \& Park, W. Tunable optical metamaterial based on liquid crystal-gold nanosphere composite. Opt. Express 17, 19459-19469 (2009).

14 Shenhar, R. \& Rotello, V. M. Nanoparticles: scaffolds and building blocks. Acc. Chem Res. 36, 549-561 (2003).

15 Zhang, S., Kou, X., Yang, Z., Shi, Q., Stucky, G. D., Sun, L., Wang, J. \& Yan, C. Nanoneckles assembled from gold rods, spheres, and bipyramids. Chem. Commun. 1816-1818 (2007).

16 Lim, I. I. S., Ip, W., Crew, E., Njoki, P. N., Mott, D., Zhong, C. J., Pan, Y. \& Zhou, S Homocysteine-mediated reactivity and assembly of gold nanoparticles. Langmuir 23, 826-833 (2007)

17 Han, L., Luo, J., Kariuki, N., Maye, M. M., Jones, V. W. \& Zhong, C. J. Novel interparticle spatial properties of hydrogen-bonding mediated nanoparticle assembly. Chem. Mater. 15, 29-37 (2003).

18 Carroll, J. B., Frankamp, B. L. \& Rotello, V. M. Self-assembly of gold nanoparticles through tandem hydrogen bonding and polyoligosilsequioxane (POSS)-POSS recognition processes. Chem. Commun. 1892-1893 (2002).

19 Zheng, W., Maye, M. M., Leibowitz, F. L. \& Zhong, C. J. Imparting biomimetic ion-gating recognition properties to electrodes with a hydrogen-bonding structured core-shell nanopartricle network. Anal. Chem. 72, 2190-2199 (2000).

20 Lim, I. I. S., Mott, D., Ip, W., Njoki, P. N., Pan, Y., Zhou, S. \& Zhong, C. J. Interparticle interactions of glutathione mediated assembly of gold nanoparticles. Langmuir 24, 8857-8863 (2008)

21 Sidhaye, D. S., Kashyap, S., Sastry, M., Hotha, S. \& Prasad, B. L. V. Gold nanoparticle networks with photoresponsive interparticle spacings. Langmuir 21 7979-7984 (2005).
22 Liu, J., Yan, L., Wang, J., Li, T., Zhao, H., Li, L., Lincoln, S. F., Prud'homme, R. \& Guo $\mathrm{X}$. Reversible photo-responsive vesicle based on the complexation between azobenzene contained molecule and $\alpha$-cyclodextrin. RSC Adv. 5, 32846-32852 (2015).

23 Callari, F., Petralia, S. \& Sortino, S. Highly photoresponsive monolayerprotected gold clusters by self-assembly of a cyclodextrinazobenzene-derived supramolecular complex. Chem. Commun. 1009-1011 (2006).

24 Akiyama, H., Tamada, K., Nagasawa, J., Abe, K. \& Tamaki, T. Photoreactivity in selfassembled monolayers formed from asymmetric disulfides having para-substituted azobenzenes. J. Phys. Chem. B 107, 130-135 (2003).

25 Jeoung, E. \& Rotello, V. M. Photochemical control of molecular recognition on selfassembled monolayer-protected gold clusters. J. Supramol. Chem. 2, 53-55 (2002).

26 Manna, A., Chen, P. -L., Akiyama, H., Wei, T. -X., Tamada, K. \& Knoll, W. Optimized photoisomerization on gold nanoparticles capped by unsymmetrical azobenzene disulfides. Chem. Mater. 15, 20-28 (2003).

27 Zhang, J., Whitesell, J. K. \& Fox, M. A. Photoreactivity of self-assembled monolayers of azobenzene or stilbene derivatives capped on colloidal gold clusters. Chem. Mater. 13 2323-2331 (2001).

$28 \mathrm{Hu}$, J., Zhang, J., Liu, F., Kittredge, K., Whitesell, J. K. \& Fox, M. A. Competitive photochemical reactivity in a self-assembled monolayer on a colloidal gold cluster. J. Am. Chem. Soc. 123, 1464-1470 (2001).

29 Ipe, B. I., Mashima, S. \& Thomas, G. Light induced modulation of self-assembly on spiropyran capped Au nanoparticle: a potential system for the controlled release of amino acid derivatives. J. Am. Chem. Soc. 125, 7174-7175 (2003).

30 Itoh, H., Naka, K. \& Chujo, Y. Photochemical assembly of gold nanoparticles utilizing the photodimerization of thymine. Langmuir 20, 1972-1976 (2004).

31 Miyoshi, E., Naka, K., Tanaka, K., Narita, A. \& Chujo, Y. Preparation of clusters having various interparticle distances based on imidazolium-modified gold nanoparticles via anion exchange. Colloids Surf. A 390, 126-133 (2011).

32 Wakselman, M., Cerutti, I. \& Chany, C. Nitrobenzyl esters as potential conjugated alkylating and differentiation promoting agents: antitumor effect in vivo. Eur. J. Med. Chem. 25, 519-526 (1990).

33 Naka, K., Narita, A., Tanaka, H., Chujo, Y., Morita, M., Inubushi, T., Nishimura, I. Hiruta, J., Shibayama, H., Koga, M., Ishibashi, S., Seki, J., Kizaka-Kondoh, S. \& Hiraoka, M. Biomedical applications of imidazolium cation-modified iron oxide nanoparticles. Polym. Adv. Technol. 19, 1421-1429 (2008)

34 Narita, A., Naka, K. \& Chujo, Y. Facile control of silica shell layer thickness on hydrophilic iron oxide nanoparticles via reverse micelle method. Colloids Surf. A 336 , 46-56 (2009).

35 Tanaka, K., Ohashi, W., Okada, H. \& Chujo, Y. Production of three radical cations from a single photon using a photo acid generator. Tetrahedron Lett. 55, 1635-1659 (2014).

36 Okada, H., Tanaka, K., Ohashi, W. \& Chujo, Y. Photo-triggered molecular release based on auto-degradable polymer-containing organic - inorganic hybrids. Bioorg. Med. Chem. 22, 3435-3440 (2014).

37 Tanaka, K., Yamane, H., Yoshii, R. \& Chujo, Y. Efficient light absorbers based on thiophenefused boron dipyrromethene (BODIPY) dyes. Bioorg. Med. Chem. 21, 2715-2719 (2013).

Supplementary Information accompanies the paper on Polymer Journal website (http://www.nature.com/pj) 